\title{
Reflective Imaging Optics Using Concave and Convex Mirrors for a Compact and Achromatic Full-field X-ray Microscope.
}

\author{
$\underline{\text { Jumpei Yamada }}^{1, *}$, Satoshi Matsuyama ${ }^{1}$, Kentaro Hata $^{1}$, Raita Hirose ${ }^{2}$, Yoshihiro Takeda ${ }^{2}$, \\ Yoshiki Kohmura ${ }^{3}$, Makina Yabashi ${ }^{3}$, Kazuhiko Omote ${ }^{2}$, Tetsuya Ishikawa ${ }^{3}$, and Kazuto Yamauchi ${ }^{1}$ \\ 1. Department of Precision Science and Technology, Graduate School of Engineering, Osaka University, \\ 2-1 Yamada-Oka, Suita, Osaka 565-0871, Japan. \\ 2. X-Ray Research Laboratory, Rigaku Corp., 3-9-12 Matsubara-cho, Akishima, Tokyo 196-8666, Japan. \\ 3. SPring-8/RIKEN, 1-1-1 Kouto, Sayo, Hyogo 679-5198, Japan. \\ * Corresponding author, yamada@up.prec.eng.osaka-u.ac.jp
}

The key component of a full-field microscope is the optical device used for imaging samples. In the Xray region, refractive lenses and zone plates have been commonly used as objectives, which can achieve high spatial resolution. In contrast to these refractive and diffractive optics, total-reflection mirrors have seldom been employed for full-field X-ray microscopy, despite possessing high reflectivity and achromaticity. One of the main reasons for this seems to be that mirror optics based on single-reflection, such as Kirkpatrick-Baez (KB) mirrors, cannot form a wide-field image due to comatic aberration. To avoid such aberrations, advanced KB (AKB) mirrors [1] or KB amélioré [2] optics have been proposed as realistic hard-X-ray imaging mirrors. These optical systems consist of four concave mirrors, which eliminate comatic aberration by satisfying the Abbe's sine condition. Consequently, these mirrors have been used for achromatic full-field X-ray microscopy [3]. However, AKB systems lack versatility, as it is difficult to obtain sufficiently large magnification factors in a compact setup. This is especially the case in the sequentially crossed-mirror configuration, such as that in KB and AKB systems, where the mirrors closer to the focal point prevents the focal length of the other mirrors from being short. To overcome this problem, an AKB mirror system consisting of two pairs of concave and convex mirrors (concave-convex AKB) was proposed $[4,5]$. In the proposed system, short effective focal lengths can be attained by shifting the principal plane. In this study, we newly design and develop an imaging mirror system for a compact and achromatic full-field X-ray microscope.

Figure 1 demonstrates a geometrical arrangement of the system designed using the parameters listed in Table 1. To obtain a sufficiently large magnification factor and wide field-of-view (FoV), we employed a conventional and a concave-convex AKB system for the vertical and horizontal directions, respectively. The length between the object and the image plane was set to approximately $2 \mathrm{~m}$, in order to mimic typical applications in X-ray analysis and laboratory-sized sources. Thanks to the concaveconvex AKB system, the magnification factor could be adjusted to be nearly equal in each direction. The incident glancing angles at the centre of mirrors were less than $4 \mathrm{mrad}$, which would provide high reflectivity up to X-ray energies of $\sim 15 \mathrm{keV}$ with an Ir or Ru coating. The numerical aperture of the imaging system was $1.0 \times 10^{-3}$ which leads a spatial resolution of $40 \mathrm{~nm}$ at $15 \mathrm{keV}$. The expected FoV of $10 \times 18 \mu \mathrm{m}^{2}$ was confirmed using our wave-optical simulator, based on Fresnel-Kirchhoff's diffraction integral.

We successfully fabricated the mirrors using our developed surface processing and measurement methods. Each mirror possessed a shape accuracy of less than $2 \mathrm{~nm}$ peak-to-valley. As reported previously [3], we should fabricate immobilized mirror pairs for the stability and practical usability of the microscope. Our previously developed monolithic-type conventional AKB mirror had elliptical and 
hyperbolic shapes on a single substrate (see Figure 2). In concave-convex AKB system, two mirrors are arranged opposite to each other, so that they were bonded to another substrate via ultraviolet curing resin. Future work lies in testing the imaging performance of this new system.

Table. 1. Parameters of the imaging mirror system. The FoV was calculated using our simulator.

\begin{tabular}{ccccc} 
& \multicolumn{2}{c}{ Vertical Imaging Mirrors } & \multicolumn{2}{c}{ Horizontal Imaging Mirrors } \\
\hline Shape & $\begin{array}{c}\text { Hyperbolic } \\
\text { concave }\end{array}$ & $\begin{array}{c}\text { Elliptical } \\
\text { concave }\end{array}$ & $\begin{array}{c}\text { Elliptical } \\
\text { concave }\end{array}$ & $\begin{array}{c}\text { Hyperbolic } \\
\text { convex }\end{array}$ \\
Mirror length $(\mathrm{mm})$ & 18.8 & 33.6 & 65.8 & 38.0 \\
Incident glancing angle $(\mathrm{mrad})$ & 3.57 & 2.87 & 3.99 & 2.10 \\
Numerical aperture & \multicolumn{2}{c}{$1.0 \times 10^{-3}$} & \multicolumn{2}{c}{$1.0 \times 10^{-3}$} \\
Field-of-view $(\mu \mathrm{m})$ & \multicolumn{2}{c}{10} & & \multicolumn{2}{c}{18} \\
Magnification factor & \multicolumn{2}{c}{42} & & 45 \\
\hline
\end{tabular}

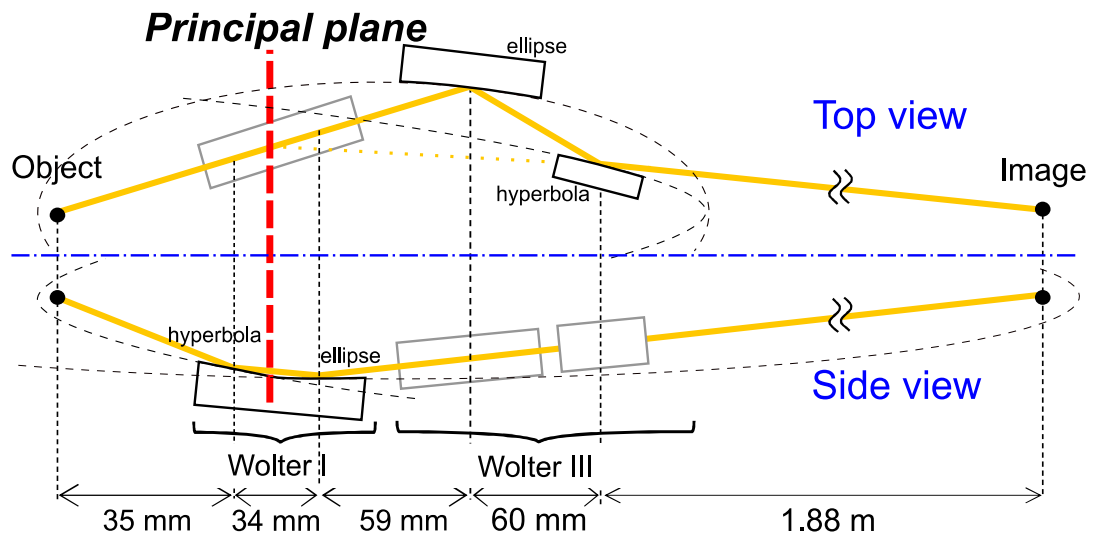

Figure. 1. Geometrical arrangement of the designed imaging mirror system.

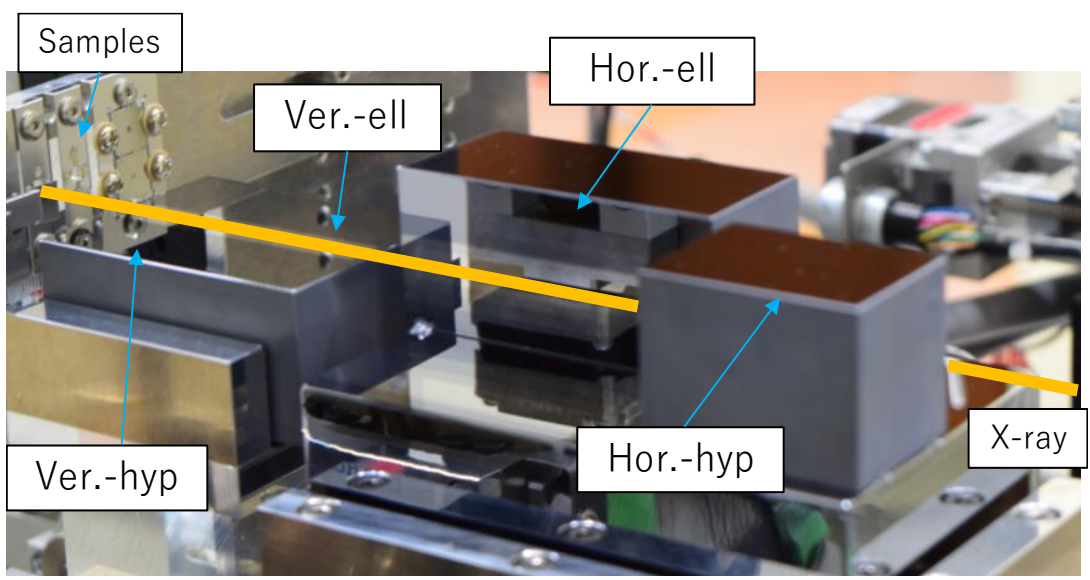

Figure. 2. Photograph of the developed imaging mirrors.

References:

[1] R. Kodama et al., Opt. Lett. 21, 1321 (1996).

[2] R. Sauneuf et al., Rev. Sci. Instrum. 68, 3421 (1997).

[3] S. Matsuyama et al., Sci. Rep. 7, 46358 (2017).

[4] J. Yamada et al., Appl. Opt. 56(4), 967 (2017).

[5] J. Yamada et al., Proc. SPIE 10386, 10386C (2017). 